\title{
Local coherence and the temporal development of second harmonic emission
}

\author{
D L Andrews and L Dávila Romero \\ School of Chemical Sciences, University of East Anglia, Norwich, NR4 7TJ, UK
}

Received 20 November 2000, in final form 27 February 2001

\begin{abstract}
In a variety of mesoscopically disordered systems, high levels of optical excitation resulting from pulsed laser irradiation can establish optical coherence within separate particles or locally ordered domains, leading to second harmonic emission whose temporal signature characterizes the decay of the excited state population. Examples of such systems will include colloids, cell and membrane suspensions, and many plastics, glasses and other modern materials. With pulsed excitation of sufficient intensity to elicit the onset of saturation, second harmonic emission on the throughput of a subsequent probe beam exhibits a characteristic decay and recovery. Detailed calculations show that such features arise not only in systems whose optical response involves two discrete levels, but also in systems of considerably greater electronic complexity. Deconvolution of the temporal trace of the harmonic signal serves as an independent means of monitoring the decay of the excited state. The extent of recovery in the harmonic signal also serves to register the extent of local coherence, and hence in many systems the localization of structural order. Finally, the principles introduced in the theory are shown to be applicable to other types of system such as certain photochromic materials.
\end{abstract}

\section{The emergence of second harmonic signals from randomly oriented systems}

In recent years the field of nonlinear optics has substantially outgrown the narrow confines of its original largely solid state domain. Increasingly attention is being focused on disordered systems, small-particle composites and fluids, where nonlinear optical processes offer novel means of material characterization [1-5]. Such characterization is generally based on the fact that these systems exhibit forms of optical nonlinearity which are very different from those of crystalline solids. For example, only a weak, incoherent, non-directed, second harmonic signal can emerge from any truly isotropic system - and then only if the constituent particles are non-centrosymmetric [6]. Although a host of exceptions to this symmetry condition have been listed [7], they generally entail conditions resulting in the removal of local isotropy-or, at very high intensities, six-wave interactions [8-11]. By exploiting the general principle, however, the process of second harmonic generation (SHG) has become a highly valuable tool for studying the surfaces of liquids and other centric systems, since any 
coherent second harmonic emission generally carries information only from the surface and not the bulk.

Against this background, the experimental detection of strongly directed SHG signals from photosynthetic bacterial membranes, randomly oriented in aqueous suspension [12,13], at first appeared to violate the condition. The paradox was resolved when it was shown that the unusually strong signal detected from isotropic suspensions, notably of purple membrane material, is attributable to optical coherence within the separate particles of the suspension [14]. As such, the harmonic emission displays an amalgam of the characteristics associated with full coherence (second harmonic generation) and incoherence (hyper-Rayleigh scattering). The analysis of angularly resolved measurements of the second harmonic under various polarization conditions led to experimental verification of this interpretation [15], which also supports occurrence of the phenomenon in locally ordered domains within disordered solids.

The coherent addition of second harmonic signals, which can only occur in regions of local order, is consistent with the local additivity of hyperpolarizabilities associated with different optical centres or chromophores. This leads to intriguing possibilities for materials strongly pumped by an ultrafast source. Here, the key feature is the relationship between the hyperpolarizabilities of optical centres in their ground and electronic excited states, under resonance conditions $[16,17]$. As a consequence of this relationship, the effective secondorder susceptibility of each domain or particle proves to be very strongly influenced by the instantaneous degree of optical excitation, and the harmonic signal acquires a temporal signature which can faithfully register the dynamics of optical excitation and decay. Thus, where a significant degree of optical excitation is established in such a system by a primary laser source, studying the characteristics of the second harmonic generated by a probe beam offers the means for direct measurement of the excited state population dynamics, and a means of gauging the extent of localized structural order.

\section{The contribution of permanent dipole moments}

To begin, it is useful to establish the significance in harmonic generation processes of permanent electric dipole moments, well illustrated by studies on the purple membrane $[18,19]$. Two of the features that have been identified as significantly enhancing the SHG process in the purple membrane are associated with the electronic properties of the principal optical centre, bacteriorhodopsin, whose chromophore comprises retinal bound to lysine via a protonated Schiff base [20]. The spectral response with bacteriorhodopsin in its K or L electronic state centres on approximately $550-610 \mathrm{~nm}$, close to resonance with the second harmonic of the input laser radiation $(1064 \mathrm{~nm}$ in the cited studies $[12,13,15,21]$. The signal therefore benefits from near-resonance amplification and is dominated by two-level behaviour-that is the first significant feature. Secondly, it has been shown that under such circumstances, SHG may be driven by a hyperpolarizability that is dependent upon the difference between the static electronic dipole moments of the resonant and ground states (see below) - and, in fact, the retinal chromophore has an unusually large difference between these permanent dipole moments. A doubly enhanced signal thus results from the combination of a resonant transition and the large dipole moment shift exhibited by the chromophore. In the theory to be developed below, these are the conditions that are assumed to apply. 


\subsection{Fluctuation dipole coupling}

In previous works $[22,23]$ it has been shown that the adoption of the following simple prescription is valid for calculation of any nonlinear optical response tensor for a two-level $(0, u)$ molecular system:

$$
\boldsymbol{\mu}^{u u} \rightarrow \boldsymbol{\mu}^{u u}-\boldsymbol{\mu}^{00}=\boldsymbol{d} \quad \boldsymbol{\mu}^{00} \rightarrow 0
$$

where $\boldsymbol{\mu}^{00}$ and $\boldsymbol{\mu}^{u u}$ are the vector ground and upper state permanent dipole moments, respectively, and the transition dipoles remain unchanged. In its rigorous basis this calculational algorithm is consistent with deployment of a 'fluctuation dipole' operator for coupling with the transverse electric (displacement) vector field $e^{\perp}$ [24]:

$$
H_{\mathrm{int}}^{\prime}=-\varepsilon_{0}^{-1}\left[\boldsymbol{\mu}-\boldsymbol{\mu}^{00}\right] \cdot \boldsymbol{e}^{\perp} .
$$

When the various time-orderings for any optical processes of interest are drawn up, application of the algorithm enables the hyperpolarizability expressions involving any connected route that entails the ground state dipole $\boldsymbol{\mu}^{00}$ to be discarded, so long as those entailing the excited state dipole $\boldsymbol{\mu}^{u u}$ are re-interpreted to invoke $\boldsymbol{d}$. The method has been explicitly validated for all elastic and inelastic processes, both degenerate and fully non-degenerate [25]. In every case its implementation leads in a matter of lines to results identical to those previously established by substantially more laborious means. It also facilitates identification of the driving role played in the optical response of many significantly polar species by the dipole difference $d$ [26-30]. In particular, it has been shown that on application of the algorithm 2.1 to second harmonic generation, the following simple expression is directly obtained for the corresponding hyperpolarizability, $\chi_{i j k}^{00}(-2 \omega ; \omega, \omega)$ :

$$
\begin{gathered}
\chi_{i j k}^{00}(-2 \omega ; \omega, \omega) \equiv \frac{\mu_{i}^{0 u} d_{j} \mu_{k}^{u 0}}{\left(E_{u 0}-2 \hbar \omega\right)\left(E_{u 0}-\hbar \omega\right)}+\frac{\mu_{k}^{0 u} d_{i} \mu_{j}^{u 0}}{\left(E_{u 0}+\hbar \omega\right)\left(E_{u 0}-\hbar \omega\right)} \\
+\frac{\mu_{j}^{0 u} d_{k} \mu_{i}^{u 0}}{\left(E_{u 0}+\hbar \omega\right)\left(E_{u 0}+2 \hbar \omega\right)}
\end{gathered}
$$

where $E_{u 0}=E_{u}-E_{0}$. Equation (2.3) represents the tensor mediating transition between the following initial and final states of the system:

$$
\begin{aligned}
& |i\rangle=\left|0 ; n, n^{\prime}\right\rangle \\
& |f\rangle=\left|0 ;(n-2),\left(n^{\prime}+1\right)\right\rangle .
\end{aligned}
$$

Here the first symbol within each ket denotes the electronic state of the nonlinear optical centre, next the number of photons of pump radiation of frequency $\omega$, and finally the number of second harmonic photons, frequency $\omega^{\prime}$. It has been shown [31] that the direct dependence of the right-hand side of equation (2.3) on the difference between the excited and ground state static electric dipole moments, $\boldsymbol{d}$, affords a useful basis for spectroscopic estimation of the hyperpolarizability through measurement of solvatochromic shifts.

In considering the parametric optical response of strongly, resonantly pumped systems, it is important to take into account that part of the response mediated by species whose initial and final states are the populated upper level $|u\rangle$. For this case a more natural choice for the transformed interaction Hamiltonian is

$$
H_{\mathrm{int}}^{\prime \prime}=-\varepsilon_{0}^{-1}\left[\boldsymbol{\mu}-\boldsymbol{\mu}^{u u}\right] \cdot \boldsymbol{e}^{\perp} .
$$

Here the algorithm given in equation (2.1) is modified by interchanging the labels 0 and $u$ :

$$
\boldsymbol{\mu}^{00} \rightarrow \boldsymbol{\mu}^{00}-\boldsymbol{\mu}^{u u}=-\boldsymbol{d} \quad \boldsymbol{\mu}^{u u} \rightarrow 0 .
$$


This has the effect of reversing the sign of $\boldsymbol{d}$ and also the energy difference $E_{u 0}$, wherever each appears, though the Hermiticity of the dipole operator ensures that for non-degenerate states the transition dipole suffers no change:

$$
\begin{aligned}
& \boldsymbol{d}=\boldsymbol{\mu}^{u u}-\boldsymbol{\mu}^{00} \rightarrow-\boldsymbol{d}=-\left(\boldsymbol{\mu}^{u u}-\boldsymbol{\mu}^{00}\right) \\
& E_{u 0}=E_{u}-E_{0} \rightarrow-E_{u 0}=E_{0 u} \\
& \boldsymbol{\mu}^{0 u}=\boldsymbol{\mu}^{u 0}
\end{aligned}
$$

For the process of second harmonic conversion mediated by a species in its upper level $u$, the initial and final system states are described in quantum electrodynamical terms by

$$
\begin{aligned}
& |i\rangle=\left|u ; n, n^{\prime}\right\rangle \\
& |f\rangle=\left|u ;(n-2),\left(n^{\prime}+1\right)\right\rangle
\end{aligned}
$$

and the expression for the corresponding hyperpolarizability tensor is closely related to its ground state counterpart: adopting the algorithm (2.6), we obtain simply

$$
\begin{gathered}
\chi_{i j k}^{u u}(-2 \omega ; \omega, \omega)=-\left\{\frac{\mu_{i}^{u 0} d_{j} \mu_{k}^{0 u}}{\left(E_{0 u}-2 \hbar \omega\right)\left(E_{0 u}-\hbar \omega\right)}+\frac{\mu_{k}^{u 0} d_{i} \mu_{j}^{0 u}}{\left(E_{0 u}+\hbar \omega\right)\left(E_{0 u}-\hbar \omega\right)}\right. \\
\left.+\frac{\mu_{k}^{u 0} d_{j} \mu_{i}^{0 u}}{\left(E_{0 u}+\hbar \omega\right)\left(E_{0 u}+2 \hbar \omega\right)}\right\} .
\end{gathered}
$$

From the result it is evident that the algorithms given in equations (2.1) and (2.6) are equivalent for SHG, as indeed they are for any three-wave mixing process. The expression for $\chi_{i j k}^{u u}(-2 \omega ; \omega, \omega)$ could have been obtained by simply interchanging the labels $u$ and 0 in equation (2.3).

In the equations for the harmonic signal it is the index-symmetrized form of the hyperpolarizability, $\chi_{i(j k)} \equiv \frac{1}{2}\left(\chi_{i j k}+\chi_{i k j}\right)$, which is necessarily invoked because of the corresponding symmetry in the radiation tensor (reflecting the equivalence of the two input photons). By inspection of the above results (equations (2.3) and (2.9)) it can be concluded that the symmetric parts of these tensors which mediate SHG are connected by the simple 'mirror' identity

$$
\chi_{i(j k)}^{00}(-2 \omega ; \omega, \omega)=-\chi_{i(j k)}^{u u}(-2 \omega ; \omega, \omega)
$$

a result which holds exactly for a discrete two-level system. For real systems with more than two levels and finite linewidths, the model will require correction to allow properly for dispersion effects. These issues are to be discussed in section 4 .

\section{Optically excited small particles}

\subsection{Harmonic emission from particles in suspension}

Let us now consider a fluid or a mesoscopically disordered material (or any other optically isotropic system) within which there are small particles (or other local domains) possessing a microscopically ordered structure. Examples of such systems include colloids, cell and membrane suspensions, and many plastics, glasses and other modern materials. It is to be assumed that each unit (particle or domain) includes a significant number of optical centres with strong optical dispersion at the frequency of the pump laser radiation or its harmonic. The purple membrane suspension studies referred to earlier [12,13,15] involved systems of exactly this type. For generality, consider any such system comprising $M$ randomly oriented 
units (individually denoted below by the subscript $m$ ) each composed of $n$ discrete molecules or other optical centres (denoted by the subscript $\xi$ ). In each unit it is assumed that there is a structurally imposed orientational correlation, i.e. a significant intrinsic rigidity. Following the work of Andrews et al $[14,15]$ the intensity of harmonic generation in the absence of prior optical excitation is given by the following expression:

$$
I \approx \frac{k^{\prime 4} I_{\omega}^{2} g_{\omega}^{(2)}}{32 \pi^{2} c \varepsilon_{0}^{2}}\left|\sum_{m}^{M}\left(\chi_{(m)}^{00} \cdot \bar{e}^{\prime} \boldsymbol{e} e\right) \exp \left(\mathrm{i} \Delta \boldsymbol{k} \cdot \boldsymbol{R}_{m}\right)\right|^{2}
$$

where $k^{\prime}$ is the magnitude of the wavevector for the harmonic photon, $I_{\omega}$ is the pump irradiance and $g_{\omega}^{(2)}$ is its degree of second-order coherence, with $e$ and $e^{\prime}$ being the polarization vectors for the fundamental and harmonic, respectively. In the above expression the signal from each particle yields a phase factor associated with the scalar product between the wavevector mismatch $\Delta \boldsymbol{k}$ and $\boldsymbol{R}_{m}$, a position vector referred to the origin of an arbitrary Cartesian frame of reference with fixed orientation. Each contributory signal is also governed by an 'effective hyperpolarizability' tensor given by

$$
\chi_{(m) i j k}^{00}=\sum_{\xi}^{n} \chi_{(\xi) \lambda \mu \nu}^{00} l_{i \lambda}^{\xi} l_{j \mu}^{\xi} l_{k \nu}^{\xi} \exp \left(\mathrm{i} \Delta \boldsymbol{k} \cdot\left(\boldsymbol{R}_{\xi}-\boldsymbol{R}_{m}\right)\right) .
$$

Here the hyperpolarizability of each individual optical centre is given by $\chi_{(\xi) \lambda \mu \nu}^{00}$, and its position is given relative to the common Cartesian frame. The factor $l_{i \lambda}^{\xi}\left(l_{j \mu}^{\xi}, l_{k v}^{\xi}\right)$ is the cosine of the angle between the space fixed axis $i(j, k)$ and the molecule fixed axis $\lambda(\mu, v)$. If the particles or ordered domains are small compared with the optical wavelengths involved, then so will be the internal distances $\left(\boldsymbol{R}_{\xi}-\boldsymbol{R}_{m}\right)$, and thus in many circumstances-even in the complete lack of wavevector matching $(\Delta k=0)$ - the phase factor in equation (3.2) can often be taken as effectively unity. However, in the following we retain its explicit form for generality. Combining equations (3.1) and (3.2), the harmonic intensity can then be written as a sum of two terms $I_{1}$ and $I_{2}$ as follows, where angular brackets denote the orientational average:

$$
\begin{aligned}
& I_{1}=M D^{(2)}\left\langle\left|\chi_{(m) i j k}^{00} \bar{e}_{i}^{\prime} e_{j} e_{k}\right|^{2}\right\rangle \\
& I_{2}=\left(\eta_{M}-M\right) D^{(2)}\left|\left\langle\chi_{(m) i j k}^{00} \bar{e}_{i}^{\prime} e_{j} e_{k}\right\rangle\right|^{2}
\end{aligned}
$$

with

$$
D^{(2)}=\frac{k^{\prime 4} I_{\omega}^{2} g_{\omega}^{(2)}}{32 \pi^{2} c \varepsilon_{0}^{2}}
$$

and

$$
\eta_{M}=\left|\sum_{\xi}^{N} \exp \left(\mathrm{i} \Delta \boldsymbol{k} \cdot \boldsymbol{R}_{\xi}\right)\right|^{2}
$$

Under wavevector matching conditions, the coherence parameter [7] $\eta_{M}$ takes the value of $M^{2}$. The harmonic intensity term $I_{2}$, which represents constructive interference between different particle units, nonetheless vanishes as a result of the isotropic average-as is well known. On the other hand, since the corresponding average is conducted over the modulus square in the 'incoherent' term, $I_{1}$, this contribution persists, representing an addition of the harmonic intensities produced by different particles. As determined by equation (3.2), these 
contributions, in fact, accommodate a coherent addition of signals from the various optical centres which each particle comprises.

Results of the same form would be obtained if the system were initially strongly pumped in such a manner that each optical centre occupied the excited state $u$. From the results of the last section (cf equation (2.10)) it is evident that in the population inversion limit the corresponding unit hyperpolarizability tensor is

$$
\begin{aligned}
\chi_{(m) i j k}^{u u} & =\sum_{\xi}^{n} \chi_{(\xi) \lambda \mu \nu}^{u u} l_{i \lambda}^{\xi} l_{j \mu}^{\xi} l_{k \nu}^{\xi} \exp \left(\mathrm{i} \Delta \boldsymbol{k} \cdot\left(\boldsymbol{R}_{\xi}-\boldsymbol{R}_{u}\right)\right) \\
& =-\sum_{\xi}^{n} \chi_{(\xi) \lambda \mu \nu}^{00} l_{i \lambda}^{\xi} l_{j \mu}^{\xi} l_{k \nu}^{\xi} \exp \left(\mathrm{i} \Delta \boldsymbol{k} \cdot\left(\boldsymbol{R}_{\xi}-\boldsymbol{R}_{u}\right)\right) \\
& =-\chi_{(m) i j k}^{00} .
\end{aligned}
$$

\subsection{Signal from a strongly pumped system}

Let us now consider a system in which, prior to the input of the pump radiation responsible for the detected harmonic output, irradiation with a beam of the appropriate resonant frequency produces a significant population of the electronic level $u$ amongst the optical centres in each particle or domain. With first-order decay kinetics the probability that a certain centre $\xi$ is excited at time $t$ is given by

$$
P\left(\boldsymbol{R}_{\xi}, t\right)=P_{0}\left(\boldsymbol{R}_{\xi}\right) \exp \left(-k\left(t-t_{0}\right)\right)
$$

where $k$ is the decay constant and $P_{0}$ is the residual probability that the upper level is excited at time $t_{0}$, the time at which the pump radiation for SHG detection is applied. Particular interest below focuses on the case where $P_{0}>0.5$. Whilst both excited and ground state species are present, the two processes described previously contribute to an effective unit hyperpolarizability given by

$\chi_{(m) i j k}=\sum_{\xi}^{n}\left[\left(1-P\left(\boldsymbol{R}_{\xi}, t\right)\right) \chi_{(\xi) \lambda \mu \nu}^{00}+P\left(\boldsymbol{R}_{\xi}, t\right) \chi_{(\xi) \lambda \mu \nu}^{u u}\right] l_{i \lambda}^{\xi} l_{j \mu}^{\xi} l_{k \nu}^{\xi} \exp \left(\mathrm{i} \Delta \boldsymbol{k} \cdot\left(\boldsymbol{R}_{\xi}-\boldsymbol{R}_{m}\right)\right)$

Using equation (3.7) then gives

$\chi_{(m) i j k}=\sum_{\xi}^{n}\left[\left(1-2 P\left(\boldsymbol{R}_{\xi}, t\right)\right) \chi_{(\xi) \lambda \mu \nu}^{00}\right] l_{i \lambda}^{\xi} l_{j \mu}^{\xi} l_{k \nu}^{\xi} \exp \left(\mathrm{i} \Delta \boldsymbol{k} \cdot\left(\boldsymbol{R}_{\xi}-\boldsymbol{R}_{m}\right)\right)$.

\subsection{Temporal profile}

We now show that the above results lead to a harmonic intensity which features a characteristic decay and recovery in its temporal profile. Let us assume for simplicity that the probability of initial excitation is identical for all optical centres, removing the $\boldsymbol{R}_{\xi}$ dependence of $P\left(\boldsymbol{R}_{\xi}, t\right)$. Let us also denote by $\beta$ the key molecular factor,

$$
\beta=\sum_{\xi}^{n} \chi_{(\xi) \lambda \mu \nu}^{00} l_{i \lambda}^{\xi} l_{j \mu}^{\xi} l_{k \nu}^{\xi} \bar{e}_{i}^{\prime} e_{j} e_{k} \exp \left(\mathrm{i} \Delta \boldsymbol{k} \cdot\left(\boldsymbol{R}_{\xi}-\boldsymbol{R}_{m}\right)\right)
$$

Again for simplicity assuming CW pump radiation, the time dependence of the harmonic emission as given by equations (3.3), (3.8), (3.10) and (3.11) takes the biexponential form

$$
I \sim|\beta|^{2}\left[1-4 \exp \left(-k t^{\prime}\right)+4 \exp \left(-2 k t^{\prime}\right)\right]
$$




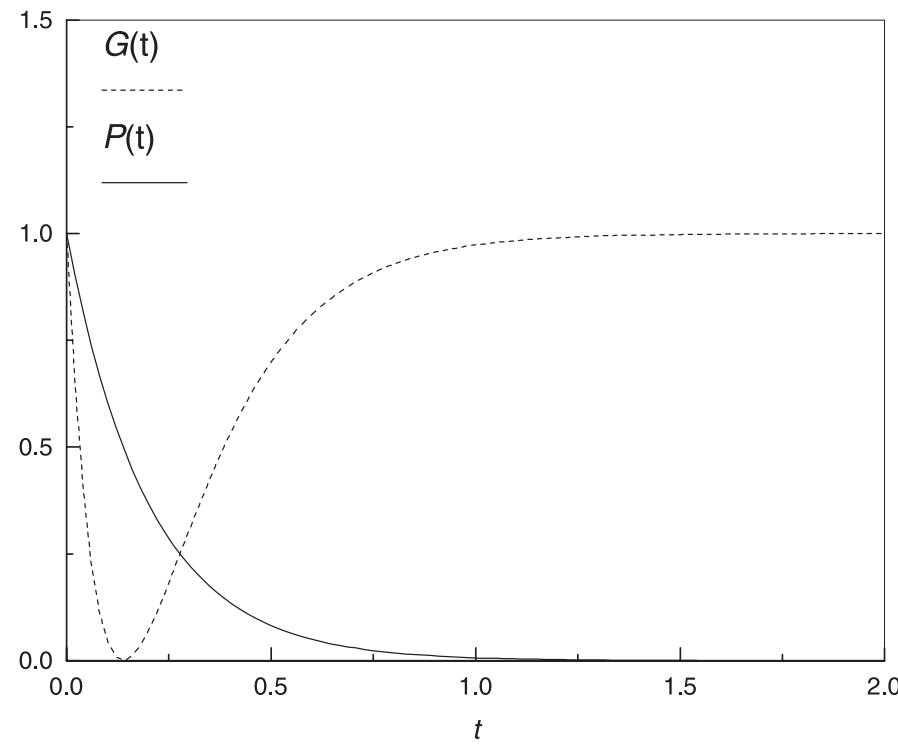

Figure 1. The excited state decay function $P(t)$, and the harmonic profiles $G(t)$.

where $t^{\prime}=t-t_{0}$ and $P_{0}\left(\boldsymbol{R}_{\xi}\right) \equiv 1$, giving as a result a trace of the form $G(t)=$ $1-4 \exp (-k t)+4 \exp (-2 k t)$, shown in figure 1 .

\section{Beyond the discrete two-level model}

To observe precisely the time dependence in the second harmonic output as described above would require the satisfaction of certain criteria detailed below. Nonetheless it is important to note at the outset that these conditions are largely a reflection of the simple two-level model employed, and in the following we show why it can be anticipated that the major features of the result will be manifest in real systems of considerably greater electronic complexity. We can then consider the experimental utility of measuring this behaviour.

First, returning to the two-level description, it is clear that a necessary condition for observation of a fall and recovery of the harmonic output as illustrated in figure 1 is the creation of a transient population inversion by the preceding excitation laser pulse. The minimum output intensity is obtained at the time where the fractional population of the upper level $u$ has fallen to exactly 0.5 , matching the ground state population. In practice, achieving initial population inversion is likely to require that the excitation pulse populates a higher level $h$ which rapidly decays to $u$, the latter playing the role of a population bottleneck as in conventional laser action. Although this emphasizes the fact that a two-level representation of the electronic energy levels is necessarily incomplete - as indeed it generally is — the inclusion of $h$ and any other levels in the electronic structure of the optical centres will not significantly affect the results, provided that those levels are no longer significantly populated once the pump for harmonic emission is applied. The harmonic signal will still be dominated by generation in centres in either the 0 or $u$ state. Other electronic levels will certainly play the role of virtual states in the hyperpolarizabilities of each of those levels, adding a background contribution to each.

The effect of involving other levels in the calculations, and also allowing for finite state linewidths, is now assessed by considering what modifications to the preceding theory ensue. 
(a)

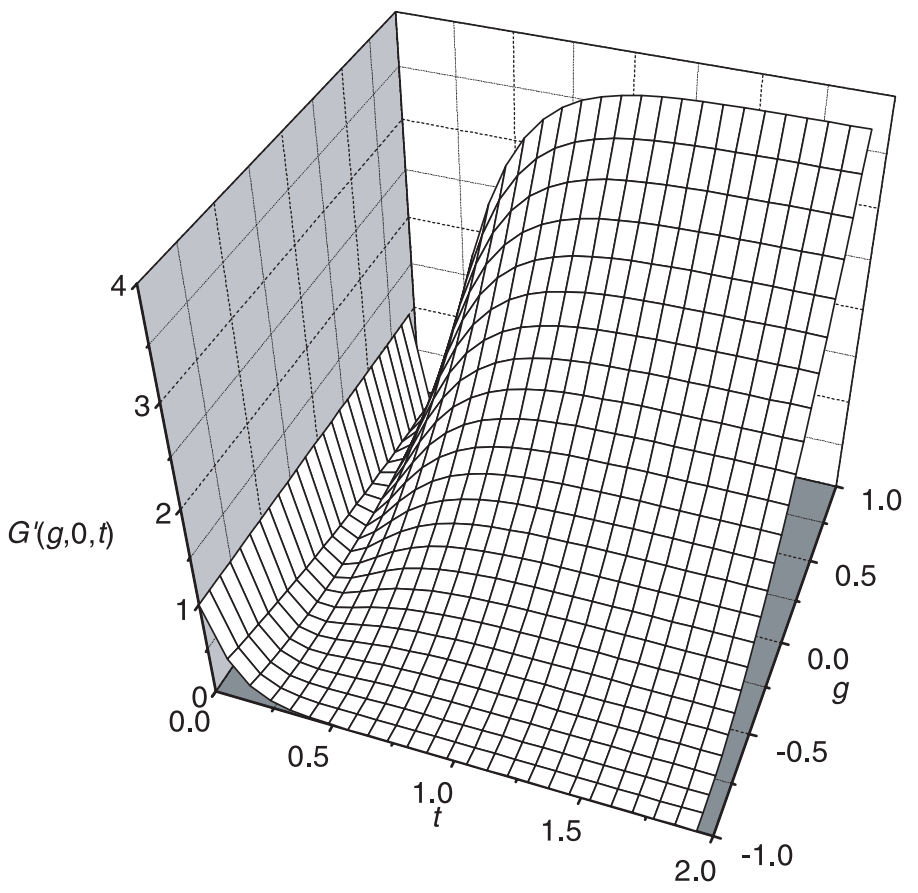

(b)

$G^{\prime}(0, h, \mathrm{t})$

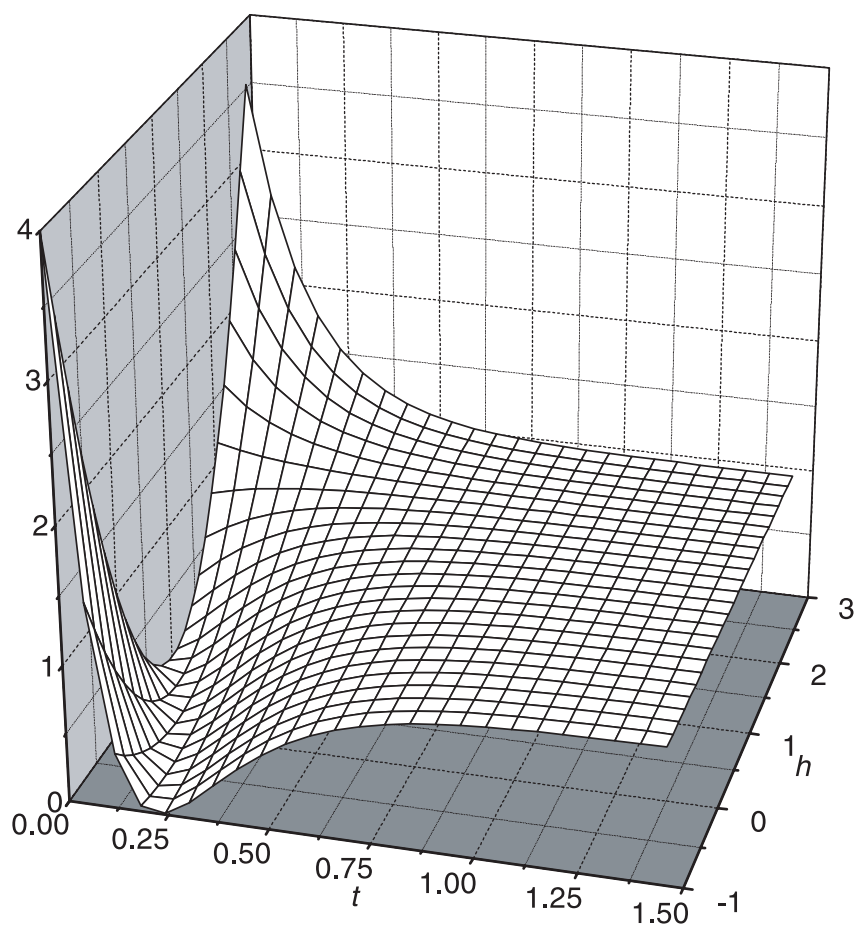

Figure 2. (a) Graph of $G^{\prime}(g, h=0, t)$ for $-1 \leqslant g \leqslant 1$ and $0 \leqslant t \leqslant 5$; (b) $G^{\prime}(g=0, h, t)$ for $-1 \leqslant h \leqslant 3$ and $0 \leqslant t \leqslant 5$. 


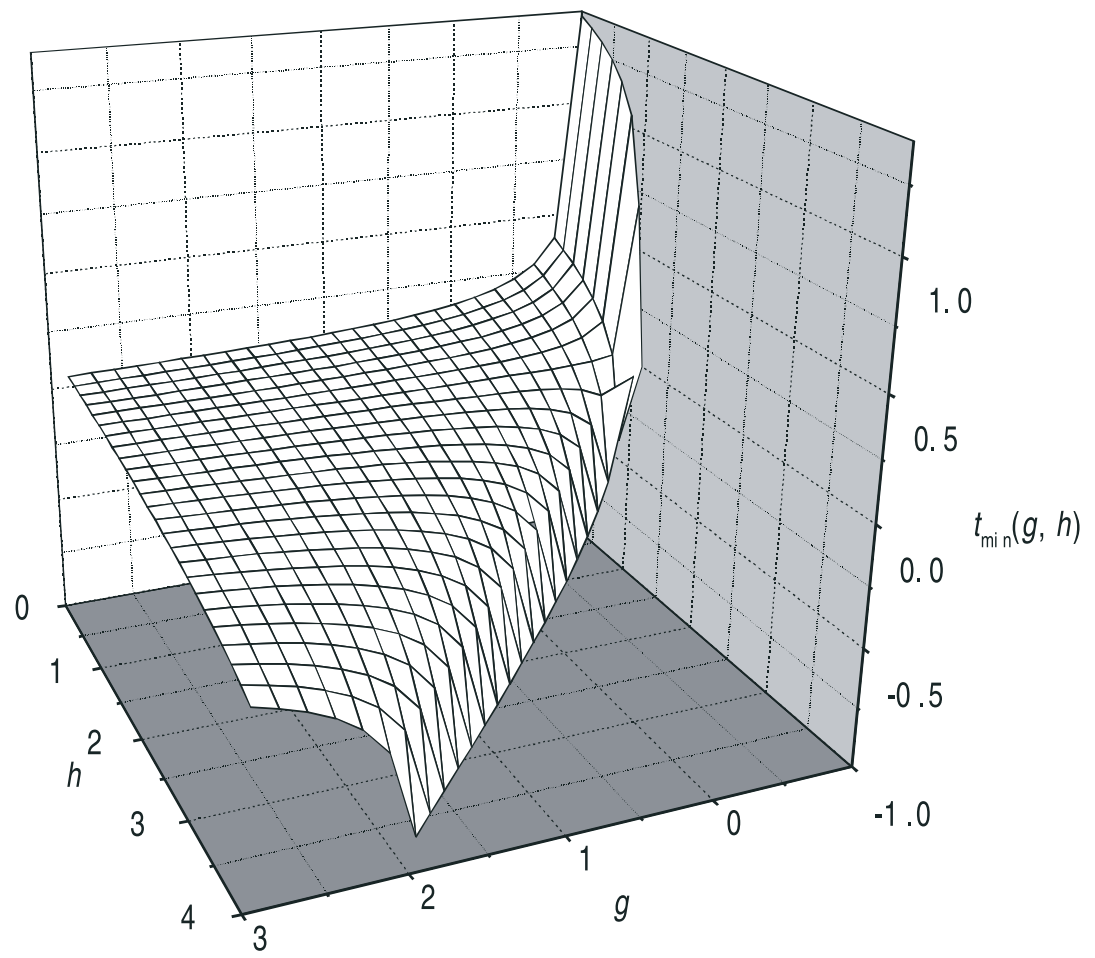

Figure 3. The variation of $t_{\min }$ in terms of $g$ and $h$.

Homogeneous linewidths can be associated with each electronic excited state through the usual incorporation of phenomenological damping factors, conferring a complex character to the hyperpolarizability tensor. It is also well known that the consideration of higher electronic states in the perturbation theoretic calculation results in the effective addition to the hyperpolarizability of a background term [7]. The net effect of such extensions to the theory is therefore the acquisition by both the tensors $\chi^{00}$ and $\chi^{u u}$ of additional (and different) background contributions, also complex, such that

$$
\begin{aligned}
& \sum_{\xi}^{n} \chi_{(\xi) \lambda \mu \nu}^{00} l_{i \lambda}^{\xi} l_{j \mu}^{\xi} l_{k \nu}^{\xi} \bar{e}_{i}^{\prime} e_{j} e_{k} \exp \left(\mathrm{i} \Delta \boldsymbol{k} \cdot\left(\boldsymbol{R}_{\xi}-\boldsymbol{R}_{m}\right)\right) \rightarrow \beta+\tilde{\beta} \\
& \sum_{\xi}^{n} \chi_{(\xi) \lambda \mu \nu}^{u u} l_{i \lambda}^{\xi} l_{j \mu}^{\xi} l_{k \nu}^{\xi} \bar{e}_{i}^{\prime} e_{j} e_{k} \exp \left(\mathrm{i} \Delta \boldsymbol{k} \cdot\left(\boldsymbol{R}_{\xi}-\boldsymbol{R}_{m}\right)\right) \rightarrow-\beta+\check{\beta} .
\end{aligned}
$$

This results in a second harmonic intensity with a more intricate time dependence of the form

$$
I \sim\left|(\beta+\tilde{\beta})-(2 \beta+\tilde{\beta}-\tilde{\beta}) \exp \left(-k t^{\prime}\right)\right|^{2}
$$

which no longer factorizes out the temporal profile $G\left(t^{\prime}\right)$. To proceed further with the analysis, we now impose certain conditions on the corrections $\tilde{\beta}$ and $\breve{\beta}$, assuming that they are subject to the simple prescription $\tilde{\beta}=g \beta, \breve{\beta}=h \beta$, where $g$ and $h$ are small (real, positive or negative) numerical factors. In this case the harmonic intensity takes the simple form:

$$
I \sim|\beta|^{2}|(1+g)-(2+g-h) \exp (-k t)|^{2} \equiv|\beta|^{2} G^{\prime}(g, h, t) .
$$


The function $G^{\prime}(g, h, t)$ has been obtained by considering that the probability $P(t)$ that a certain centre $\xi$ is excited at time $t$ is given by equation (3.8) with $t_{0}=0$, consistent with negligible residual excitation of higher energy levels at that time.

Figures 2( $a$ ) and $(b)$ portray the temporal development function $G^{\prime}(g, h, t)$ for $h=0$ and $g=0$, respectively. The harmonic intensity expression, equation (4.3), leads to a minimum in the harmonic emission at a time given by

$$
t_{\min }(g, h)=k^{-1} \ln \left(\frac{2+g-h}{1+g}\right) .
$$

From this result it emerges that the minimum exists only for certain values of the parameters $g$ and $h$, specifically under the conditions $2+g-h>0$ and $1+g>0$ or $2+g-h<0$ and $1+g<0$. The former pair of conditions will hold under normal circumstances, corresponding to upper and lower level hyperpolarizabilities dominated by a two-level response, where $|h|<1$ and $|g|<1$. However, although we are particularly concerned with such cases, others can be considered. Figure 3 shows over a wider range of values how $t_{\min }$ varies with $h$ and $g$, clearly exhibiting where the divergence appears.

\section{Discussion}

In the light of the results presented above it is useful to recall that the behaviour we have identified, in the second harmonic profile of a system of randomly oriented small particles or ordered domains in complex materials, owes its origin to the local coherence between harmonic emission processes at different optical centres within each particle or domain. This behaviour is dominated by features associated with a two-level optical response but, provided the pump or harmonic frequencies are close to resonance, broadly similar effects are anticipated in systems of considerably greater electronic complexity. In this connection, there are also potential applications in monitoring processes of molecular self-assembly, developing principles which have been established for this purpose on the basis of incoherent (hyper-Rayleigh) second harmonic scattering $[32,33]$. The characteristic signal recovery which we have identified is the key feature, and determination of the biexponential form of the harmonic profile will faithfully register the dynamics of excited state decay.

The same principles which engineer the distinctive decay and revival of second harmonic emission in strongly pumped two-level systems should also operate in photochromic materials, in some cases under substantially less demanding conditions. Here the key factor is a photochemically induced interconversion between two molecular forms which have hyperpolarizabilities of opposite signs. A good example is afforded by spiropyran and photomerocyanine $\left(\beta\right.$ values of $1.9 \times 10^{-30}$ esu and $-43.0 \times 10^{-30}$ esu, respectively [34]), which interconvert through the application of UV or UV/visible radiation. Clearly, optical coherence within locally ordered domains (for example, in a suspension of polymer particles) where both species are present during the relatively long timescale $\left(10^{2} \mathrm{~s}\right)$ for interconversion should lead to a dip in the SHG signal at a time corresponding to $\sim 4.4 \%$ photomerocyanine. Given the current interest in the use of such materials for optical memories and switches [35,36], experimental characterization of the effect will be of considerable significance.

Finally, we note that the interpretation of results from systems of the kind we have described, which include colloids, cell and membrane suspensions, plastics, glasses and many other modern materials, will have structural as well as kinetic implications. The local coherence, responsible for the partial cancellation of the harmonic signal after a characteristic delay time, is entirely dependent on a structural rigidity within each particle. This need not mean that all the optical centres are identically aligned, but that they do not rotate significantly 
with respect to each other (at least over the timescale for the harmonic measurements). The extent of recovery in the harmonic signal serves to register the extent of local coherence, and hence in many systems the localization of structural order. In any less than completely rigid system, such as for example in the purple membrane studies which prompted the current analysis, it might be possible to assess the degree of local flexibility from the extent of harmonic recovery. In photobiological materials, this could hold intriguing possibilities for the study of protein folding.

\section{References}

[1] Shalaev V M, Poliakov E Y and Markel V A 1996 Phys. Rev. B 532437

[2] Shalaev V M, Poliakov E Y, Markel V A and Botet R 1997 Physica A 241249

[3] Shalaev V M, Markel V A, Poliakov E Y, Armstrong R L, Safonov V P and Sarychev A K 1998 J. Nonlinear Opt. Phys. Mater. 7131

[4] Clays K, Hendrickx E, Verbiest T and Persoons A 1998 Adv. Mater. 10643

[5] Shalaev V M 2000 Nonlinear Optics of Random Media (Springer Tracts in Modern Physics vol 158) (Berlin: Springer)

[6] Andrews D L 1993 J. Mod. Opt. 40939

[7] Andrews D L 1993 Modern Nonlinear Optics Part 2, ed M W Evans and S Kielich (Adv. Chem. Phys. vol 85) p 545

[8] Andrews D L 1994 Nonlin. Opt. 825

[9] Allcock P and Andrews D L 1997 J. Phys. B: At. Mol. Opt. Phys. 303731

[10] Hands I D, Lin S J, Meech S R and Andrews D L 1998 J. Chem. Phys. 10910580

[11] Lin S, Hands I D, Andrews D L and Meech S R 1999 J. Phys. Chem. A 1033830

[12] Song Q, Wan C and Johnson C K 1994 J. Phys. Chem. 981999

[13] Schmidt K and Rayfield G W1994 Appl. Opt. 334286

[14] Andrews D L, Allcock P and Demidov A A 1995 Chem. Phys. 1901

[15] Allcock P, Andrews D L, Meech S R and Wigman A J 1996 Phys. Rev. A 532788

[16] Heflin J R, Rodenberger D C, Shi R F, Wu M, Wang N Q, Cai Y M and Garito A F 1992 Phys. Rev. A 45 R4233

[17] Rodenberger D C, Heflin J R and Garito A F 1992 Nature 359309

[18] Henderson R, Baldwin J M, Ceska T A, Zemlin F, Beckmann E and Dowing K H 1990 J. Mol Biol. 213899

[19] Birge R R 1981 Ann. Rev. Biophys. Bio. 10315

[20] Oesterhelt D and Stoeckenius W 1971 Nature New Biol. 233149

[21] Wan C, Qian J and Johnson C K1991 Biochemistry 302664

[22] Andrews D L, Dávila Romero L C and Meath W J 1999 J. Phys. B: At. Mol. Opt. Phys. 321

[23] Dávila Romero L C and Andrews D L 1999 J. Phys. B: At. Mol. Opt. Phys. 322277

[24] Bishop D M 1994 J. Chem. Phys. 1006535

[25] Dávila Romero L C 1999 PhD Thesis University of East Anglia

[26] Oudar J L and Chemla D S 1977 J. Chem. Phys. 662664

[27] Dick B and Hohlneicher G 1982 J. Chem. Phys. 765755

[28] Meath W J and Power E A 1984 J. Phys. B: At. Mol. Phys. 17763

[29] Kmetic M A and Meath W J 1985 Phys. Lett. A 108340

[30] Andrews D L and Meath W J 1993 J. Phys. B: At. Mol. Opt. Phys. 264633

[31] Paley M S, Harris J M, Looser H, Baumert J C, Bjorklund G C, Jundt D and Tweig R J 1989 J. Org. Chem. 54 3774

[32] Clays K, Hendrickx M, Triest M, Verbiest T, Persoons A, Dehu C and Bredas J L 1993 Science 2621419

[33] Ray P C and Das P K 1997 Chem. Phys. Lett. 281243

[34] Atassi Y, Delaire J A and Nakatani K 1995 J. Phys. Chem. 9916320

[35] Berkovic G, Krongauz V and Weiss V 2000 Chem. Rev. 1001741

[36] Delaire J A and Nakatani K 2000 Chem. Rev. 1001817 